\title{
Evaluation of self-absorption of manganese emission lines in Laser Induced Breakdown Spectroscopy measurements
}

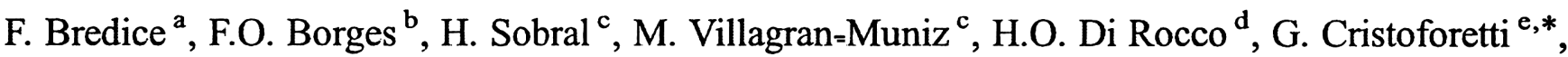

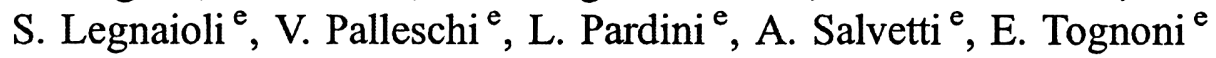 \\ a Centro de Investigaciones Opticas, P.O. Box 124, (1900) La Plata, Argentina \\ b Instituto de Física, Universidade Federal Fluminense, UFF, Campus da Praia Vermelha-Gragoatá, 24210-340 Niterói, Rio de Janeiro, Brazil \\ c Laboratorio de Fotofisica, Centro de Ciencias Aplicadas y Desarrollo Tecnológico, Universidad Nacional Autónoma de México, \\ Apartado Postal 70-186 México Distrito Federal, P. O. Box 04510, Mexico \\ 'Instituto de Física "Arroy'o Seco", Facultad de Ciencias Exactas, U.N.C.P.B.A., Pinto 399, 7000 Tandil, Argentina \\ ' Applied Laser Spectroscopy Laboratory, IPCF-CNR, Area della Ricerca di Pisa, Via G. Moruzzi 1, 56124 Pisa, Italy
}

Received 26 January 2006; accepted 29 October 2006

\begin{abstract}
This paper is part of a more general study aimed to the determination of the best experimental procedures for reliable quantitative measurements of Fe-Mn alloys by LIBS. In this work, attention is pointed on the self-absorption processes, whose effect deeply influences the LIBS measurements, reflecting in non-linear calibration curves. The effect of self-absorption on the line intensity can be quantified by defining a self-absorption coefficient, that measures the deviation of the line intensity from the linear extrapolation of the curve of growth in the optically thin regime. The authors demonstrated in a previous paper that self-absorption coefficients could be calculated once the electron density of the plasma is known and the Stark coefficients of the lines are available. However, when the Stark coefficients of the lines of interest are not known, a different approach is needed. In this work a new method for evaluation of self-absorption coefficients in LIBS measurements is presented, which does not require the knowledge of Stark coefficients. In order to understand the basic principles and setting out the theoretical tools that will be used for the analysis of the alloys, a preliminary study was done on pure Mn; LIBS spectra were acquired in different experimental conditions, at different laser energies and different delays after the laser irradiation of the sample. Moreover, collinear double pulse measurements were also performed. Analytical relations were derived and experimental procedures devised for evaluation of the self-absorption coefficients of several Mn lines, which are important for characterization and control of the experimental conditions in which the analysis is performed.
\end{abstract}

(C) 2006 Elsevier B.V. All rights reserved.

\section{Introduction}

In recent years, the Laser-Induced Breakdown Spectroscopy (LIBS) technique affirmed as a powerful analytic tool for the determination of the elemental composition of materials [1-4]. Due to its intrinsic fastness of analysis, limited dimensions of the experimental setup and no need for sample pre-treatment, LIBS has been proposed and used for in-situ and on-line analysis of materials even in hostile environments characterized

\footnotetext{
Abbreviations: LIBS; Fe-Mn alloys; Self-absorption.

* Corresponding author. Tel.: +39050 3152222; fax: +39050 3152230 .

E-mail address: gabriele@ipcf.cnr.it (G. Cristoforetti).
}

by high temperatures $[5,6]$ or radiation hazards $[7,8]$, in the presence of toxic wastes $[9,10]$ or in potentially harmful situations [11]. The analysis of industrial materials, as ferrous and non-ferrous alloys has also attracted the attention of many researchers, for the potential use of LIBS in closed-loop diagnostic systems and as a non-invasive, minimally destructive method capable to operate as a fast screening technique $[6,12-$ 16]. In this framework, it seems particularly interesting to see the use of LIBS for the analysis of Fe-Mn alloys, which are extremely important, for example, in shape-memory alloys applications [17-21].

However, a precise compositional analysis of these alloys by LIBS requires a clear understanding of the main effects 
influencing the LIBS spectrum, and in particular self-absorption effects [22-28] and matrix effects [29-31]. In this work, the self-absorption effects on pure manganese will be studied, and the theoretical basis of the spectral analysis will be laid.

In a previous paper [28] it was demonstrated that the selfabsorption effect on spectral line intensity can be calculated once the electron density of the plasma is known and the Stark coefficients of the lines are available. Unfortunately, in literature the measured Stark coefficients are available only for a few ionic Mn lines [32]; moreover the measured values differ substantially from the calculated analogues [33]. Therefore, a different approach is needed. The method here proposed is based on the measurement of the intensity ratio of two lines of the same ionization stage and on the comparison of the experimental to the theoretically predicted value; this approach is well known in the literature as a direct check of the absence of important self-absorption effects in spectroscopic measurements [34]. In a recent paper [35], Amamou et al. used the ratio of emission lines coming from the same multiplet for correcting the self-absorption effects in the measurements of transition probabilities of silicon lines, in the limit of moderate self-absorption. The analysis of emission lines belonging to several multiplets is also the basis of the semiempirical method developed by Friedjung and Muratorio [36,37].

In this paper, starting from the theoretical treatment of these effects, we propose a method for quantifying the influence of selfabsorption on the measured intensity of the lines considered, at the same time providing a quick way of determining, among the different emission lines of a given element, the ones more appropriate for the calculation of plasma parameters (temperature and electron density) as well as for LIBS analytical measurements.

\section{Theoretical analysis: emission from a homogeneous plasma rod}

According to the two-levels treatment of atomic emission [38], the light intensity, i.e. the energy emitted per unit time, unit surface and unit wavelength ( $\mathrm{erg} \mathrm{s}^{-1} \mathrm{~cm}^{-3}$ ) by a homogeneous plasma rod of length $l$ can be calculated writing the so-called transport equation, which describes the change in radiation intensity after travelling a distance $d x$ into the rod, in the form

$d I(\lambda, x)=\varepsilon(\hat{\lambda}) d x-\kappa(\lambda) I(\lambda, x) d x$

where $\varepsilon(\lambda)$ represents the plasma spontaneous emissivity, i.e. the energy emitted per unit time, unit volume and unit wavelength (erg s-1 $\mathrm{cm}^{-4}$ ):

$\varepsilon\left(\lambda_{)}\right)=\frac{h c}{4 \pi \lambda_{0}} A_{\mathrm{ki}} n_{\mathrm{k}} L(\hat{\lambda})$

$h$ is the Planck constant (erg s), $c$ is the speed of light ( $\mathrm{cm} \mathrm{s}^{-1}$ ), $\lambda_{0}$ is the central wavelength of the transition $(\mathrm{cm}), A_{\mathrm{ki}}$ represents the transition probability between the upper level $k$ and the lower level $i\left(\mathrm{~s}^{-1}\right), n_{\mathrm{k}}$ is the population $\left(\mathrm{cm}^{-3}\right)$ of the upper level and $L$ $(\lambda)$ is the spectral emission profile $\left(\mathrm{cm}^{-1}\right)$.

The $\kappa(\lambda)$ coefficient is the absorption coefficient $\left(\mathrm{cm}^{-1}\right)$, which takes into account both the absorption by lower level atoms and the stimulated emission of the upper level atoms under the effect of the radiation $I(\lambda, x)$ :

$\kappa(\lambda)=\frac{\hat{i}_{0}^{4}}{8 \pi c} A_{\mathrm{ki}} g_{\mathrm{k}} \frac{n_{\mathrm{i}}}{g_{\mathrm{i}}}\left(1-\frac{n_{\mathrm{k}} g_{\mathrm{i}}}{g_{\mathrm{k}} n_{\mathrm{i}}}\right) L(\hat{\lambda})$

where $g_{\mathrm{k}}$ and $g_{\mathrm{i}}$ are the degeneracies of the upper and lower level (dimensionless), respectively, and $n_{\mathrm{i}}$ is the population $\left(\mathrm{cm}^{-3}\right)$ of the lower level.

Since in typical LIBS experiments the detectors used do not give the intensity of the emission, but a measure of the number of photons emitted (per second) at a given wavelength, Eq. (1) should be divided by the energy of the photon $h c / \lambda_{0}$ for obtaining the transport equation for the photon number $n_{\mathrm{p}}$, yielding:

$\begin{aligned} d n_{\mathrm{p}}(\lambda, x) & =\frac{\varepsilon\left(\lambda_{0}\right)}{h c} \lambda_{0} d x-\kappa(\hat{\lambda}) n_{\mathrm{p}}(\lambda, x) d x \\ & =\varepsilon^{\prime}(\lambda) d x-\kappa(\hat{\lambda}) n_{\mathrm{p}}(\hat{\lambda}, x) d x\end{aligned}$

where $\varepsilon^{\prime}(\lambda)$ is defined as $\varepsilon^{\prime}(\lambda) \equiv \frac{1}{4 \pi} A_{\mathrm{ki}} n_{\mathrm{k}} L(\hat{\lambda})$.

The solution of Eq. (4) gives the number of photons emitted by a homogeneous plasma rod of length $l$, in the form

$n_{\mathrm{p}}(\lambda, l)=\frac{\varepsilon^{\prime}(\lambda)}{\kappa(\lambda)}\left(1-e^{-\kappa(\lambda) l}\right)$

Assuming the Local Thermal Equilibrium in the plasma rod, the population of the energy level $k, i$ can be expressed through the Boltzmann distribution of equilibrium:

$n_{k, i}=g_{k, i} n \frac{e^{-\frac{E_{k, i}}{k_{\mathrm{B}} T}}}{U(T)}$

where $n$ is the total number of atoms of the species per unit volume $\left(\mathrm{cm}^{-3}\right), E_{k, i}$ is the energy of the level (erg), $k_{\mathrm{B}}$ is the Boltzmann constant (erg $\mathrm{K}^{-1}$ ), $T$ is the plasma temperature $(K)$ and $U(T)$ is the partition function for the species (dimensionless).

Therefore, in this approximation

$\varepsilon^{\prime}(\lambda)=\frac{1}{4 \pi} A_{k i} g_{\mathrm{k}} n \frac{e^{-\frac{E_{\mathrm{k}}}{k_{\mathrm{B}} T}}}{U(T)} L(\lambda)$

and

$\kappa(\lambda)=\frac{\lambda_{0}^{4}}{8 \pi c} A_{k i} g_{\mathrm{k}} n \frac{e \frac{E_{\mathrm{i}}}{k_{\mathrm{B}} T}}{U(T)} L(\lambda)$

where the further approximation $\frac{n_{\mathrm{k}} g_{\mathrm{i}}}{g_{k} n_{\mathrm{i}}}<<1$ was introduced, corresponding to neglecting the stimulated emission effect with respect to plasma absorption.

\subsection{Self-absorption}

In order to quantify the effect of self-absorption on the emission lines intensity, let us introduce the self-absorption coefficient SA, defined as the ratio of the actual intensity of the emission line at its maximum $\left(n_{\mathrm{p}}\left(\lambda_{0}\right)\right.$, in counts per seconds) over the value $\left(n_{\mathrm{p}_{0}}\left(\lambda_{0}\right)\right)$ obtained by extrapolating the curve of 
growth valid in the optically thin regime to the same emitters number density of the actual measurement [27]:

$\mathrm{SA}=\frac{n_{\mathrm{p}}\left(\lambda_{0}\right)}{n_{\mathrm{p}_{0}}\left(\lambda_{0}\right)}=\frac{\left(1-e^{-\kappa\left(\lambda_{0}\right) l}\right)}{\kappa\left(\lambda_{0}\right) l}$.

The number density of the emitters is in general not known and therefore the actual value of SA cannot be directly calculated; notwithstanding, the definition given in expression (9) is useful for deriving the expected behaviour of the line intensity in correspondence of change in the global plasma parameters (size, total density, electron density and temperature).

In a previous paper [28], it was demonstrated that the ratio of the integral intensity of the self-absorbed emission line $N_{\mathrm{p}}$ over the non self-absorbed one $N_{\mathrm{p}_{\mathrm{o}}}$ (defined by extrapolation as in expression 9) scales as

$\frac{N_{\mathrm{p}}}{N_{\mathrm{p}_{0}}}=\frac{\int n_{\mathrm{p}}(\lambda) d \lambda}{\int n_{\mathrm{p}_{0}}(\lambda) d \lambda}=(\mathrm{SA})^{\beta}$

with $\beta=0.44$.

Bearing in mind that in typical laser-induced plasmas the Stark effect is the dominant line broadening mechanism, while the Doppler effect can be safely neglected, we can assume that the emission lineshape $L(\lambda)$ has a normalized Lorentzian shape

$L(\lambda)=\frac{\Delta \lambda_{0}}{4\left(\lambda-\lambda_{0}\right)^{2}+\Delta \hat{\lambda}_{0}^{2}}$

In this case, it has been demonstrated [28] that the FWHM of the measured emission lines becomes

$\Delta \lambda=\Delta \lambda_{0}(\mathrm{SA})^{\alpha}$

with $\alpha=-0.56$.

The equations here reported are the basic tools that we will use in the following for the interpretation of the experimental data.

\subsection{Intensity ratio of two lines}

According to Eq. (10), and taking into account the definition of SA coefficient (Eq. (9)), the intensity ratio of two lines of the same species can be written as:

$\frac{\left(N_{\mathrm{p}}\right)_{2}}{\left(N_{\mathrm{p}}\right)_{1}}=\frac{\left(N_{\mathrm{p}_{0}}\right)_{2}}{\left(N_{\mathrm{p}_{0}}\right)_{1}} \frac{(\mathrm{SA})_{2}^{\beta}}{(\mathrm{SA})_{1}^{\beta}}=\frac{\left(A_{k i} g_{\mathrm{k}} e^{-\frac{E_{\mathrm{k}}}{\mathrm{k}_{\mathrm{B}} T}}\right)_{2}}{\left(A_{k i} g_{\mathrm{k}} e^{-\frac{E_{\mathrm{k}}}{k_{\mathrm{B}} T}}\right)_{1}} \frac{(\mathrm{SA})_{2}^{\beta}}{(\mathrm{SA})_{1}^{\beta}}$

where the subscripts 1 and 2 correspond to the lines considered. In turn, the self-absorption coefficient ratio is by definition (Eq. (9))

$\frac{(\mathrm{SA})_{2}}{(\mathrm{SA})_{1}}=\frac{\kappa\left(\lambda_{0}\right)_{1}}{\kappa\left(\lambda_{0}\right)_{2}} \frac{\left(1-e^{-\kappa\left(\lambda_{0}\right)_{2} l}\right)}{\left(1-e^{-\kappa\left(\lambda_{0}\right)_{1} l}\right)}$

Moreover, from Eqs. (8) and (11) we can write

$\frac{\kappa\left(\lambda_{0}\right)_{2}}{\kappa\left(\lambda_{0}\right)_{1}}=\left(\frac{\lambda_{0}^{4} A_{k i} g_{\mathrm{k}} e^{-\frac{\varepsilon_{\mathrm{i}}}{k_{\mathrm{B}} T}}}{\Delta \lambda_{0}}\right)_{2} /\left(\frac{\lambda_{0}^{4} A_{k i} g_{\mathrm{k}} e^{-\frac{\varepsilon_{j}}{k_{\mathrm{B}}^{T}}}}{\Delta \lambda_{0}}\right)_{1}$
From Eqs. (13), (14) and (15) we can immediately see that the dependence of the line intensity ratio on the plasma parameters is quite complex, since both the absolute values of the $\kappa\left(\lambda_{0}\right)$ coefficients and their ratio depend on plasma temperature; moreover, both the absolute values of the self-absorption coefficients and their ratio depend on the plasma dimensions $l$ and, through the parameter $n$ (the total number of atoms of the species considered), on the plasma electron density $n_{\mathrm{e}}$.

In fact, the number density of the species considered obeys, under the approximation of LTE, the Saha-Boltzmann equation (which also depends on the plasma temperature) [39]

$n_{\mathrm{e}} \frac{n^{\mathrm{II}}}{n^{\mathrm{I}}}=\frac{\left(2 \pi m_{\mathrm{e}} k_{\mathrm{B}} T\right)^{3 / 2}}{h^{3}} \frac{2 U^{\mathrm{II}}(T)}{U^{\mathrm{I}}(T)} e^{-\frac{\varepsilon_{\text {ion }}}{k_{\mathrm{B}} T}}$

where $m_{\mathrm{e}}$ is the electron mass $(\mathrm{g})$ and $E_{\mathrm{ion}}$ is the ionization energy (erg) of the element.

The superscripts I and II refer, respectively, to the neutral and singly ionized species of the element under study, which in typical LIBS experiments are the only dominant species, i.e. $n^{\text {Tot }} \approx n^{\mathrm{I}}+n^{\text {II }}$ where $n^{\text {Tot }}$ is total number density of the element.

A further dependence on the electron temperature and density is brought into the equations by the line width $\Delta \lambda_{0}$, which, in first approximation (i.e. neglecting the atom and ion impact contribution to the broadening), is proportional to the electron density through the temperature-dependent Stark coefficient $w_{\mathrm{s}}(T)$ [40]

$\Delta \lambda_{0} \approx 2 w_{\mathrm{s}}(T) n_{\mathrm{e}}$

\subsubsection{Limit case: weakly self-absorbed lines}

In the limit case of low self-absorption $\left(\kappa\left(\lambda_{0}\right) l<<1\right)$ the SA coefficient defined in Eq. (9) equals unity, therefore the ratio of the integrated intensities of two lines of the same species in this limit becomes:

$\frac{\left(N_{\mathrm{p}}\right)_{2}}{\left(N_{\mathrm{p}}\right)_{1}} \approx \frac{\left(A_{k i} g_{\mathrm{k}} e^{-\frac{E_{\mathrm{k}}}{\mathrm{k}_{\mathrm{B}} T}}\right)_{2}}{\left(A_{k i} g_{\mathrm{k}} e^{-\frac{E_{\mathrm{k}}}{\mathrm{k}^{\mathrm{B}} T}}\right)_{1}} \quad \kappa_{1,2}\left(\lambda_{0}\right) l<<1$.

\subsubsection{Limit case: strongly self-absorbed lines}

Despite the intrinsic complexity of Eqs. (13)-(15), we can easily derive the SA coefficients in the limit $\kappa\left(\lambda_{0}\right) l>>1$, which corresponds to the condition of very high self-absorption. In this case

$S A \approx \frac{1}{\kappa\left(\lambda_{0}\right) l}$.

The ratio of the integrated intensities of two lines of the same species, both subjected to strong self-absorption, can be obtained by putting Eq. (19) together with the equation system (13)-(15), as

$\frac{\left(N_{\mathrm{p}}\right)_{2}}{\left(N_{\mathrm{p}}\right)_{1}} \approx \frac{\left(A_{k i} g_{\mathrm{k}} e^{-\frac{E_{\mathrm{k}}}{k_{\mathrm{B}} T}}\right)_{2}}{\left(A_{k i} g_{\mathrm{k}} e^{-\frac{E_{\mathrm{k}}}{k_{\mathrm{B}} T}}\right)_{1}}\left[\frac{\left(\frac{\lambda_{0}^{4} A_{k i} g_{\mathrm{k}} e^{-\frac{E_{\mathrm{B}}}{k_{\mathrm{B}} T}}}{\Delta \lambda_{0}}\right)_{1}}{\left(\frac{\lambda_{0}^{4} A_{k i} g_{\mathrm{k}} e^{-\frac{E_{\mathrm{B}} T}{k_{\mathrm{B}} T}}}{\Delta \lambda_{0}}\right)_{2}}\right]^{\beta} \quad \kappa_{1,2}\left(\lambda_{0}\right) l>>1$. 


\subsubsection{Limit case: lines belonging to the same multiplet}

We expect that the intensity ratio of lines connecting levels with similar $E_{\mathrm{k}}$ and $E_{\mathrm{i}}$, i.e. lines belonging to the same multiplet, would not change with the plasma temperature. From the point of view of the real measurements, if the plasma temperature was the only time-varying parameter during the plasma evolution, in this limit case the intensity ratio should be independent on the delay of acquisition of the LIBS spectra. By resorting to the previous expressions (18) and (20), the intensity ratio in this limit case should then be comprised between the two extremes

$\frac{\left(N_{\mathrm{p}}\right)_{2}}{\left(N_{\mathrm{p}}\right)_{1}}=\frac{\left(A_{k i} g_{\mathrm{k}}\right)_{2}}{\left(A_{k i} g_{\mathrm{k}}\right)_{1}} \quad \kappa_{1,2}\left(\lambda_{0}\right) l<<1$

and

$\frac{\left(N_{\mathrm{p}}\right)_{2}}{\left(N_{\mathrm{p}}\right)_{1}}=\left[\frac{\left(A_{k i} g_{\mathrm{k}}\right)_{2}}{\left(A_{k i} g_{\mathrm{k}}\right)_{1}}\right]^{(1-\beta)}\left[\frac{\left(\lambda_{0}^{4}\right)_{1}}{\left(\lambda_{0}^{4}\right)_{2}}\right]^{\beta} \quad \kappa_{1,2}\left(\lambda_{0}\right) l \gg>1$

in the limit of low and high self-absorption, respectively, since the Lorentzian width of the lines, mainly dominated by the Stark broadening effect, is the same for lines belonging to the same multiplet. Considering that, in general, $\left[\frac{\left.\left(\lambda_{0}^{\alpha}\right)\right]}{\left(\hat{\alpha}_{0}^{*}\right)_{2}}\right]^{\beta} \approx 1$ for lines be= longing to the same multiplet, we observe that the most intense line of the multiplet is always more self-absorbed than the less intense. Therefore, if the intensity ratio of two lines belonging to the same multiplet is greater than one in low self-absorption conditions, at the increase of self-absorption the ratio will decrease, and vice-versa.

\subsubsection{General case}

In the general case, according to Eq. (15), the ratio $\frac{\left(\Delta \lambda_{0}\right)_{2}}{\left(\Delta \lambda_{0}\right)_{1}}$ must be known for obtaining the predicted intensity ratio of two lines. If the Stark coefficients of the two lines are known, according to Eq. (17) the above mentioned ratio can be immediately calculated as

$$
\frac{\left(\Delta \lambda_{0}\right)_{2}}{\left(\Delta \lambda_{0}\right)_{1}} \approx \frac{\left(w_{\mathrm{s}}\right)_{2}}{\left(w_{\mathrm{s}}\right)_{1}}
$$

If the Stark coefficients of the lines are not known, on the other hand, the ratio of the Lorentzian widths of the emission lines can be derived from the experimental data combining Eqs. (12) and (13), in the form

$$
\frac{\left(\Delta \lambda_{0}\right)_{2}}{\left(\Delta \lambda_{0}\right)_{1}}=\frac{(\Delta \lambda)_{2}}{(\Delta \lambda)_{1}}\left(\frac{\left(N_{\mathrm{p}}\right)_{2}}{\left(N_{\mathrm{p}}\right)_{1}}\right)^{-\frac{\alpha}{\beta}}\left(\frac{\left(A_{k i} g_{\mathrm{k}} e^{-\frac{E_{\mathrm{k}}}{k_{\mathrm{B}} T}}\right)_{2}}{\left(A_{k i} g_{\mathrm{k}} e^{-\frac{E_{\mathrm{k}}}{k_{\mathrm{B}} T}}\right)_{1}}\right)^{\frac{\alpha}{\beta}}
$$

The knowledge of the $\frac{\left(\Delta \lambda_{0}\right)_{2}}{\left(\Delta \lambda_{0}\right)_{1}}$ ratios for the lines here considered can now be exploited for obtaining, from Eq. (15), the ratio of the $\kappa\left(\lambda_{0}\right)_{1}$ and $\kappa\left(\lambda_{0}\right)_{2}$ coefficients.
This ratio can be substituted in Eq. (13), yielding:

$$
\begin{aligned}
\frac{\left(N_{\mathrm{p}}\right)_{2}}{\left(N_{\mathrm{p}}\right)_{1}} & =\frac{\left(A_{k i} g_{\mathrm{k}} e^{-\frac{E_{\mathrm{k}}}{k_{\mathrm{B}} T}}\right)_{2}}{\left(A_{k i} g_{\mathrm{k}} e^{-\frac{E_{\mathrm{k}}}{k_{\mathrm{B}} T}}\right)_{1}}\left(\frac{\kappa\left(\lambda_{0}\right)_{1}}{\kappa\left(\lambda_{0}\right)_{2}} \frac{\left(1-e^{-\kappa\left(\lambda_{0}\right)_{2} l}\right)}{\left(1-e^{-\kappa\left(\lambda_{0}\right)_{1} l}\right)}\right)^{\beta} \\
& =a\left[\frac{b\left(1-e^{-\kappa\left(\lambda_{0}\right)_{2} l}\right)}{\left(1-e^{b \kappa\left(\lambda_{0}\right)_{2} l}\right)}\right]^{\beta}
\end{aligned}
$$

where

$a=\frac{\left(A_{k i} g_{\mathrm{k}} e^{-\frac{E_{\mathrm{k}}}{k_{\mathrm{B}} T}}\right)_{2}}{\left(A_{k i} g_{\mathrm{k}} e^{-\frac{E_{\mathrm{k}}}{k_{\mathrm{B}} T}}\right)_{1}}$

is the low self-absorption limit of the intensity ratio (Eq. (18)) and

$b=\frac{\left(\lambda_{0}^{4} A_{k i} g_{\mathrm{k}} e^{-\frac{E_{i}}{k_{\mathrm{B}} T}}\right)_{1}}{\left(\hat{\lambda}_{0}^{4} A_{k i} g_{\mathrm{k}} e^{-\frac{E_{\mathrm{i}}}{k_{\mathrm{B}} T}}\right)_{2}}\left(\frac{\left(\Delta \lambda_{0}\right)_{2}}{\left(\Delta \lambda_{0}\right)_{1}}\right)$.

Eq. (25) can be numerically solved for the opacity $\kappa\left(\lambda_{0}\right)_{2} l$ since the intensity ratio $\frac{\left(N_{\mathrm{p}}\right)_{2}}{\left(N_{\mathrm{p}}\right)_{1}}$ of the two lines, the $\frac{\left(\Delta \lambda_{0}\right)_{2}}{\left(\Delta \lambda_{0}\right)_{1}}$ parameter and the plasma temperature $T$ are known from the experimental data. Once the $\kappa\left(\lambda_{0}\right) l$ parameter is known, for a given line, at given experimental conditions, the self-absorption coefficient SA can be easily calculated from Eq. (9).

\section{Experimental results}

The Fe-Mn alloys will be analysed, using the LIBS technique, in the different laboratories participating to the SAILORMAN (Southern American-Italian LIBS-Oriented Research for Material Analysis Network), a cooperative research network connecting LIBS laboratories in Argentina (CIOP, La Plata and University of Tandil), Mexico (Universidad Nacional Autónoma de México), Brazil (Universidade Federal Fluminense) and Italy (ALS Lab.-CNR in Pisa), using the experimental facilities and the specific know-how in spectroscopy there available. For this preliminary work on pure manganese, the samples were analysed at CIOP (La Plata, Argentina) and at ALS Lab (Pisa, Italy). The CIOP LIBS setup used a Nd-YAG Laser emitting pulses at $1064 \mathrm{~nm}$ with $200 \mathrm{~mJ}$ energy and $7 \mathrm{~ns}$ FWHM, coupled to a Czerny-Turner spectrometer (50 cm focal length, 1200 grooves/ $\mathrm{mm}$ grating) equipped with an Andor iCCD for time-resolved spectral analysis. At ALS Laboratories the measurements on the same pure Mn sample were performed using Modì (Mobile DualPulse Instrument) [41], a double pulse mobile LIBS system using a Double-Pulse Nd-YAG Laser emitting pulses at $1064 \mathrm{~nm}$ with $60 \mathrm{~mJ}$ energy per pulse and $12 \mathrm{~ns}$ FWHM, coupled with an Echelle spectrometer equipped with an iCCD. The measurements in Pisa were performed both in single pulse, at two energies (60 and $120 \mathrm{~mJ})$ and double pulse regime $(60+60 \mathrm{~mJ}$ with an interpulse delay of $2 \mu \mathrm{s}$ ). In particular, the evolution of the plasma in time has been studied by varying the acquisition delay for every given irradiation regime. Fifty LIBS spectra were averaged for each of the different acquisition delay times considered, calculated after the (second) laser pulse, with a 50 ns gate in La Plata 

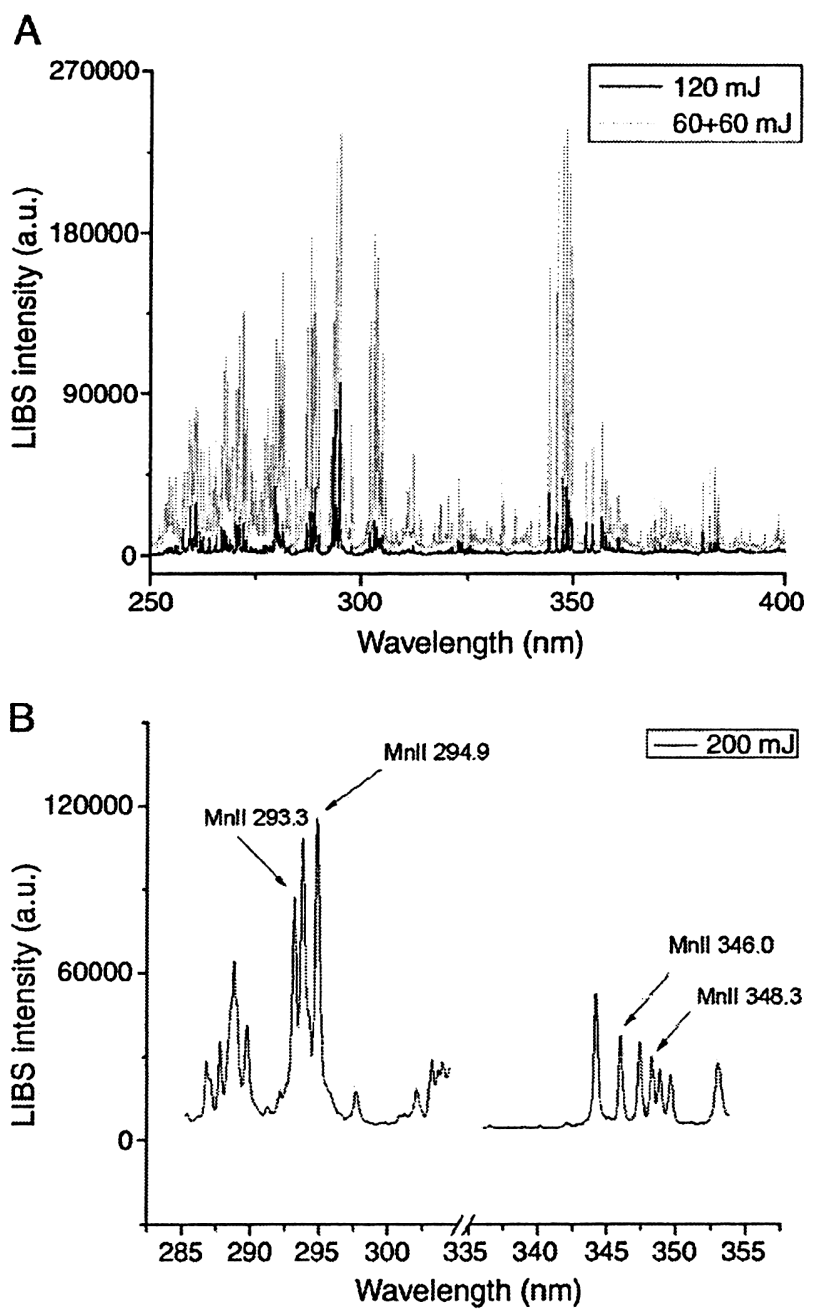

Fig. 1. Typical LIBS spectra on pure manganese obtained A) at ALS Lab. in Pisa (only a portion of the spectrum is shown, where the most intense $\mathrm{Mn}$ lines are present). Black: single pulse at $120 \mathrm{~mJ}$; Gray: Double pulse at $60+60 \mathrm{~mJ}$ with $2 \mu \mathrm{s}$ interpulse delay. B) At CIOP, La Plata (single pulse at $200 \mathrm{~mJ}$ ). The spectra shown were acquired $1 \mu \mathrm{s}$ after the laser spark. Some of spectral lines considered in this paper are marked.

measurements and $500 \mathrm{~ns}$ gate in Pisa measurements. While the measurements in Pisa were performed with a broadband spectrometer, which allowed for acquisition of the whole LIBS spectrum from 230 to $900 \mathrm{~nm}$ in a single run, the La Plata spectrometer was operated in two spectral windows between 285-304 nm and 336-354 nm, where several intense Mn II lines are well visible. A comparison between the LIBS spectra taken in Pisa and La Plata is shown in Fig. $1 \mathrm{~A}$ and $\mathrm{B}$.

In order to calculate the self-absorption coefficients of the $\mathrm{Mn}$ lines under study, the main parameters characterizing the laserinduced plasma (temperature and electron density) were measured. In particular, electron density was determined from the spectra acquired in Pisa, in both single and double pulse configuration, measuring the Stark broadening of the Hydrogen Balmer alpha at $656.3 \mathrm{~nm}[28,42]$. The use of the hydrogen lines for electron density measurements has been demonstrated to yield a precise
Table 1

Spectral parameters of the Mn lines considered in this paper

\begin{tabular}{llrrrrr}
\hline Species & Wavelength $(\mathrm{nm})$ & $g_{\mathrm{i}}$ & $g_{\mathrm{k}}$ & $E_{\mathrm{i}}\left(\mathrm{cm}^{-1}\right)$ & $E_{\mathrm{k}}\left(\mathrm{cm}^{-1}\right)$ & $A_{\mathrm{ki}}\left(\mathrm{s}^{-1}\right)$ \\
\hline Mn I & 382.4 & 6 & 6 & $1.75 \times 10^{4}$ & $4.36 \times 10^{4}$ & $2.3 \times 10^{7}$ \\
Mn I & 383.4 & 6 & 8 & $1.75 \times 10^{4}$ & $4.35 \times 10^{4}$ & $4.3 \times 10^{7}$ \\
${ }^{*}$ Mn I & 471.0 & 8 & 8 & $2.33 \times 10^{4}$ & $4.45 \times 10^{4}$ & $1.7 \times 10^{7}$ \\
*Mn I & 472.7 & 6 & 6 & $2.35 \times 10^{4}$ & $4.47 \times 10^{4}$ & $1.7 \times 10^{7}$ \\
${ }^{*}$ Mn I & 473.9 & 4 & 4 & $2.37 \times 10^{4}$ & $4.48 \times 10^{4}$ & $2.4 \times 10^{7}$ \\
Mn I & 478.5 & 8 & 8 & $1.85 \times 10^{4}$ & $3.94 \times 10^{4}$ & $4.0 \times 10^{7}$ \\
Mn I & 482.4 & 10 & 8 & $1.87 \times 10^{4}$ & $3.94 \times 10^{4}$ & $5.0 \times 10^{7}$ \\
Mn II & 261.8 & 11 & 13 & $2.76 \times 10^{4}$ & $6.58 \times 10^{4}$ & $2.9 \times 10^{8}$ \\
Mn II & 293.3 & 5 & 3 & $9.47 \times 10^{3}$ & $4.36 \times 10^{4}$ & $2.0 \times 10^{8}$ \\
Mn II & 293.9 & 5 & 5 & $9.47 \times 10^{3}$ & $4.35 \times 10^{4}$ & $1.9 \times 10^{8}$ \\
Mn II & 294.9 & 5 & 7 & $9.47 \times 10^{3}$ & $4.37 \times 10^{4}$ & $1.9 \times 10^{8}$ \\
*Mn II & 344.2 & 9 & 7 & $1.43 \times 10^{4}$ & $4.34 \times 10^{4}$ & $4.3 \times 10^{7}$ \\
*Mn II & 346.0 & 7 & 5 & $1.46 \times 10^{4}$ & $4.35 \times 10^{4}$ & $3.2 \times 10^{7}$ \\
*Mn II & 347.4 & 5 & 3 & $1.48 \times 10^{4}$ & $4.36 \times 10^{4}$ & $1.5 \times 10^{7}$ \\
*Mn II & 348.3 & 5 & 5 & $1.47 \times 10^{4}$ & $4.35 \times 10^{4}$ & $2.0 \times 10^{7}$ \\
${ }^{*}$ Mn II & 348.9 & 3 & 3 & $1.49 \times 10^{4}$ & $4.36 \times 10^{4}$ & $2.5 \times 10^{7}$ \\
\hline
\end{tabular}

The spectral lines used for plasma temperature measurements are marked with an asterisk.

determination of the plasma electron density, which is not affected by self-absorption and does not depend on any specific hypothesis of Local Thermal Equilibrium [28]. The error estimated on the electron density measurements is of the order of $10 \%$. The plasma temperature was measured using the Saha-Boltzmann plot method, described in Ref. [43]. The lines used for the determination of the temperature are shown in Table 1; all of them are relatively isolated, non-resonant emission lines. As shown in the following discussion, these lines are weakly to moderately selfabsorbed, and thus they can be considered appropriate for temperature measurements using the Saha-Boltzmann method. In this case, since a single plasma temperature can be defined only in the presence of thermal equilibrium, the hypothesis of LTE has to be introduced. As demonstrated in a previous paper [28], this procedure guarantees an accuracy in plasma temperature determination around $\pm 5 \%$, which is more than satisfactory considering the

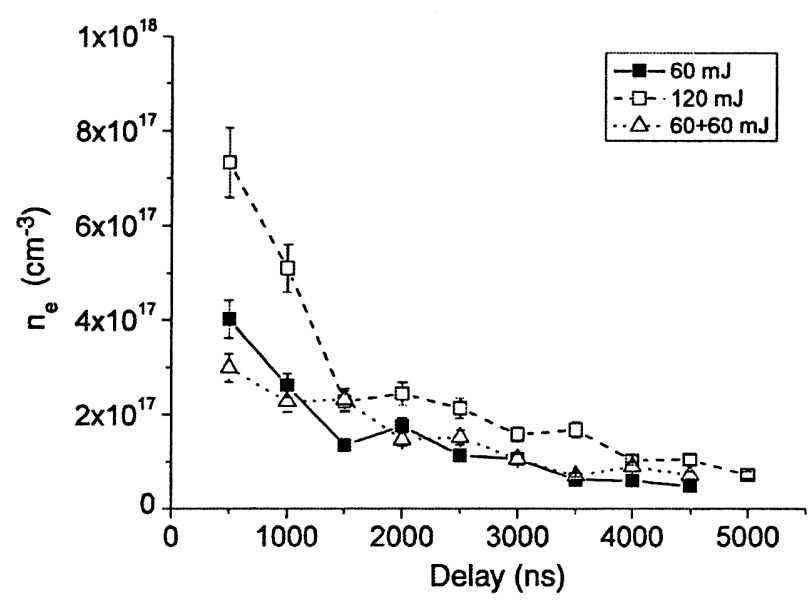

Fig. 2. Time dependence of the electron density, at $60 \mathrm{~mJ}$ laser energy (full squares), $120 \mathrm{~mJ}$ laser energy (open squares), $60+60 \mathrm{~mJ}$ with $2 \mu$ s interpulse delay (open triangles). The relative errors are of the order of $10 \%$. 


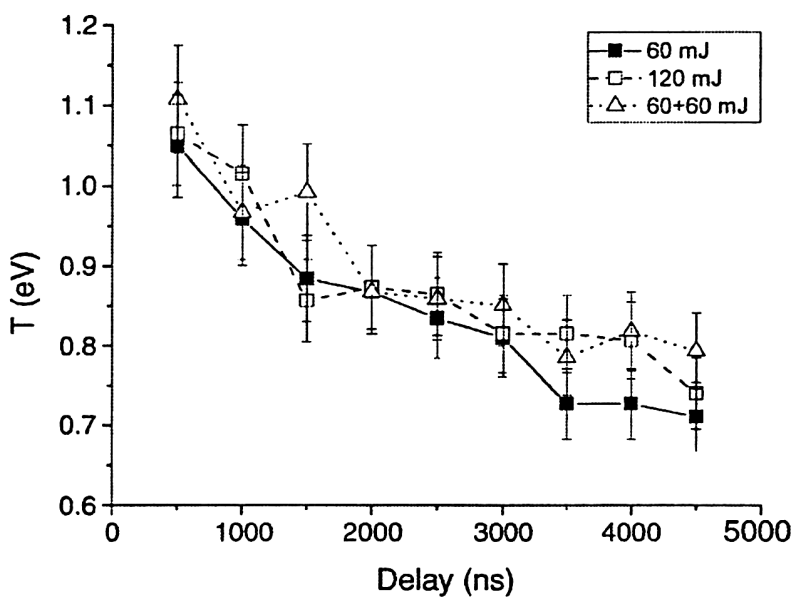

Fig. 3. Time dependence of the plasma temperature, at $60 \mathrm{~mJ}$ laser energy (full squares), $120 \mathrm{~mJ}$ laser energy (open squares), $60+60 \mathrm{~mJ}$ with $2 \mu$ s interpulse delay (open triangles). The relative errors are of the order of $5 \%$.

above discussion about LTE and the uncertainties related to the use of the approximation of homogeneous plasma (see below).

The evolution of plasma electron density and temperature is plotted in Figs. 2 and 3, as a function of the acquisition delay. In the time window investigated, the electron density varies from about $8 \times 10^{17} \mathrm{~cm}^{-3}$ to $4 \times 10^{16} \mathrm{~cm}^{-3}$. At a given delay time, the electron density corresponding to laser energy of $120 \mathrm{~mJ}$ is roughly double of the one corresponding to $60 \mathrm{~mJ}$. The electron density measured in double pulse mode $(60+60 \mathrm{~mJ}$ with a $2 \mu \mathrm{s}$ interpulse delay) is essentially the same, within the experimental errors, of the one corresponding to a single pulse of $60 \mathrm{~mJ}$. As for the plasma temperature, in the explored time window it decays from values of about $1.1 \mathrm{eV}$ to $0.7 \mathrm{eV}$.

Within the experimental errors, the plasma temperature is about the same in all the configurations studied (single pulse $60 \mathrm{~mJ}$, single pulse $120 \mathrm{~mJ}$, double pulse $60+60 \mathrm{~mJ}$ ). These results are coherent with the behaviours reported in the literature in similar conditions [44].

It is important to note, at this point, that both the evaluation of electron density through the Stark broadening of $H_{\alpha}$ line and the evaluation of the plasma temperature through the SahaBoltzmann plot method rely on the hypothesis of homogeneous plasma. However, in recent years several experimental works $[23,26]$ have shown that laser-induced plasmas in LIBS experiments are characterized by spatial variation of their main parameters (temperature and electron density) which obviously cannot be reproduced in the framework of the homogeneous plasma approximation. On the other hand, at least in the limit of small to medium self-absorption, it has been demonstrated that a realistic evaluation - and, possibly, correction - of the selfabsorption effects can be obtained using the homogeneous plasma approximation (see, for example, Gornushkin et al. [22], Bulajic et al. [27], El Sherbini et al. [28], Amamou et al. [35]). Obviously, the extension of the self-absorption theoretical treatment to the more realistic case of inhomogeneous plasma would be highly desirable; however, at the moment, it seems that only the limit situation of strong plasma inhomogeneity (leading to self-reversal effect) can be treated in a relatively easy way using space-integrated LIBS spectra (see Amamou et al. [24] and Gornuskin et al. [25], for example). A more detailed calculation of the self-absorption coefficients, taking into account the plasma inhomogeneity, calls for different and more complex experimental strategies, involving tomographic reconstruction of the plasma parameters (see for example Aguilera et al. [26], Corsi et al. [44], and Cristoforetti et al. [45]).

\subsection{Measurements on ionized Mn lines}

Using the theoretical treatment described in previous section, we have evaluated the self-absorption coefficients of 9 intense ionized Mn lines (Mn II at 261.8; 293.3; 293.9; 294.9; 344.2; $346.0 ; 347.4 ; 348.3 ; 348.9 \mathrm{~nm}$ ). These lines have been chosen since they are well insulated and free from interferences of other $\mathrm{Mn}$ lines, therefore they are good candidates for analytical LIBS measurements. The spectroscopic parameters of these lines are shown in Table 1. As an example of the application of the theoretical treatment described in previous sections, in Fig. 4 are shown the intensity ratios of the lines Mn II at 293.3/Mn II at $294.9 \mathrm{~nm}$ (lines belonging to the same multiplet) and $\mathrm{Mn} \mathrm{II}$ at 293.3/Mn II at $348.3 \mathrm{~nm}$ (lines belonging to different multiplets). The intensity ratios are plotted as a function of the acquisition delay after the laser pulse. In case of double pulse measurements, the delay is calculated after the arrival on the sample of the second laser pulse. Since the first couple of lines in Fig. 4 (Mn II at 293.3 and $\mathrm{Mn}$ II at $294.9 \mathrm{~nm}$ ) belongs to the same multiplet, the theoretical treatment of Section 2.2.3 is applicable. According to the theory, if a limit case (low or high self-absorption limit) is applicable, the intensity ratio of these two lines is essentially independent on the variation in plasma

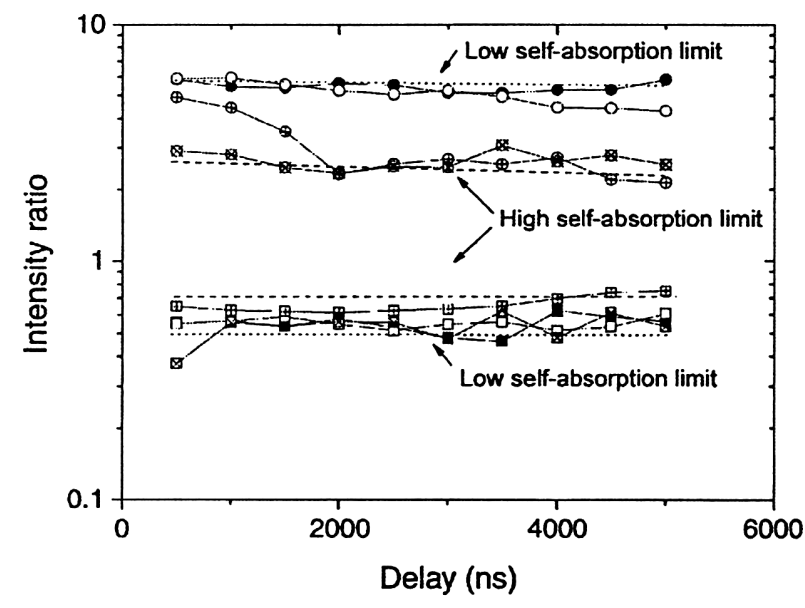

Fig. 4. Time dependence of the intensity ratios of $\mathrm{Mn}$ II at 293.3/Mn II at $294.9 \mathrm{~nm}$ lines (full squares, $60 \mathrm{~mJ}$ laser energy, open squares, $120 \mathrm{~mJ}$ laser energy, crossed squares, $200 \mathrm{~mJ}$ laser energy (La Plata measurements), diagonally crossed squares, $60+60 \mathrm{~mJ}$ double pulse measurements) and $\mathrm{Mn}$ II at 293.3/Mn II at $348.3 \mathrm{~nm}$ lines (full circles, $60 \mathrm{~mJ}$ laser energy, open circles, $120 \mathrm{~mJ}$ laser energy, crossed circles, $200 \mathrm{~mJ}$ laser energy (La Plata measurements), diagonally crossed circles, $60+60 \mathrm{~mJ}$ double pulse measurements). The dotted and dashed lines represent the low self-absorption and high self-absorption limits, respectively, for the two line pairs. Note the logarithmic scale on the $y$ axis. 


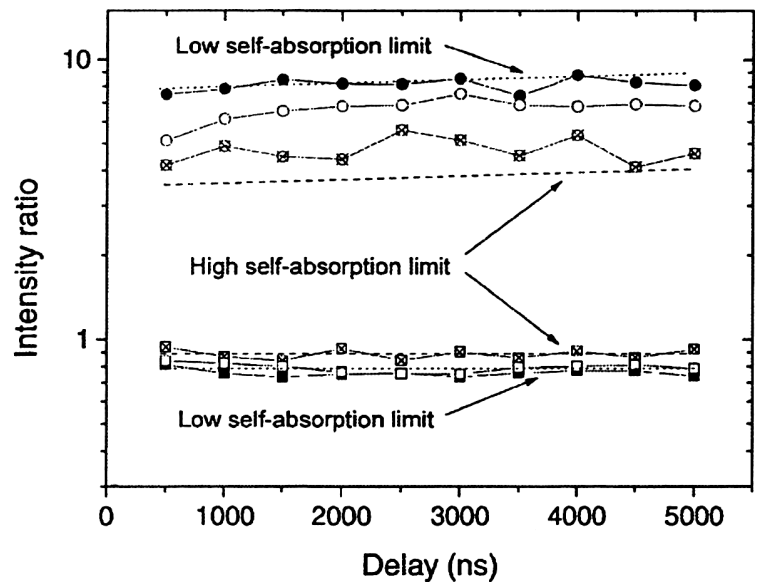

Fig. 5. Time dependence of the intensity ratios of $\mathrm{Mn} \mathrm{I}$ at $478.5 / \mathrm{Mn} \mathrm{I}$ at $482.4 \mathrm{~nm}$ lines (full squares, $60 \mathrm{~mJ}$ laser energy; open squares, $120 \mathrm{~mJ}$ laser energy; diagonally crossed squares, $60+60 \mathrm{~mJ}$ double pulse measurements) and $\mathrm{Mn} \mathrm{I}$ at $482.4 / \mathrm{Mn} \mathrm{I}$ at $472.7 \mathrm{~nm}$ lines (full circles, $60 \mathrm{~mJ}$ laser energy; open circles, $120 \mathrm{~mJ}$ laser energy; diagonally crossed circles, $60+60 \mathrm{~mJ}$ double pulse measurements). The dotted and dashed lines represent the low self-absorption and high self-absorption limits, respectively, for the two line pairs. Note the logarithmic scale on the $y$ axis.

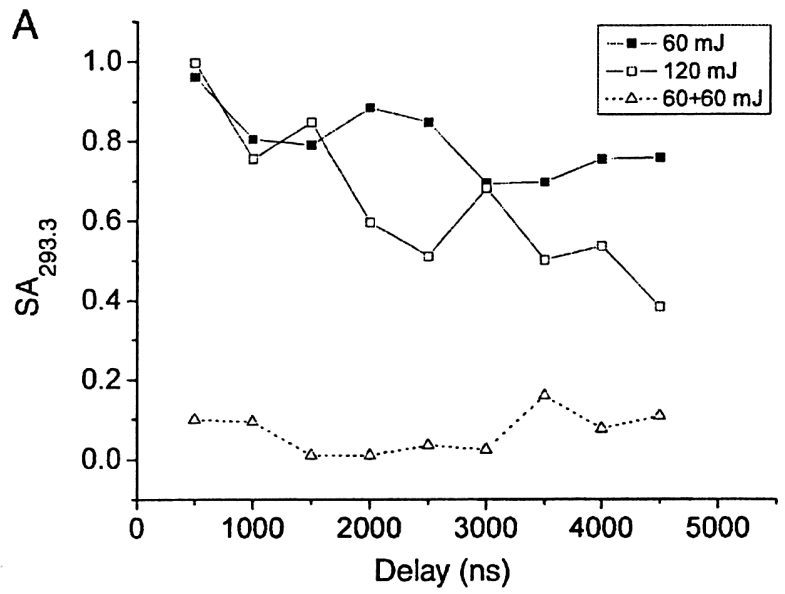

C

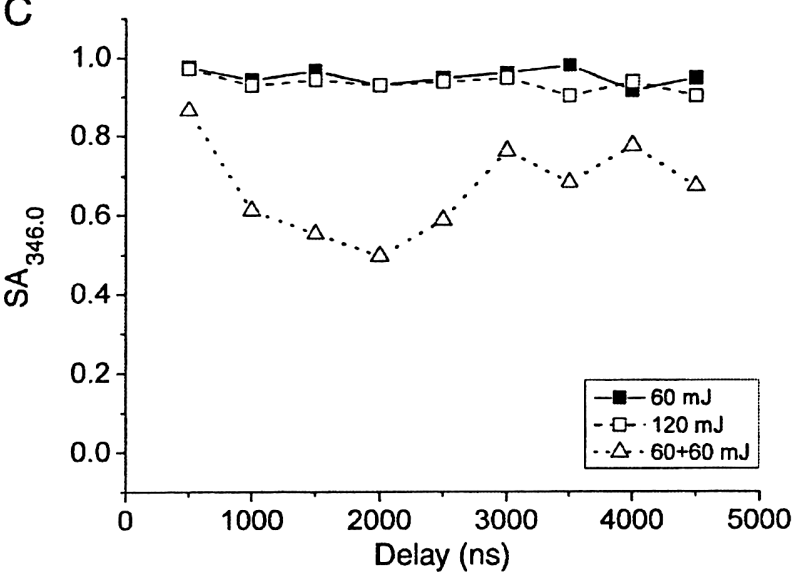

temperature and, consequently, on the increasing acquisition delay. The theoretically predicted values of the ratio are about 0.5 for the low self-absorption limit and about 0.7 for the high self-absorption limit.

Even though the two limit values are quite close, it can be noticed that single pulse irradiation leads to optically thin lines, while the double pulse irradiation shifts the plasma conditions toward the high self-absorption limit.

In the same figure, the intensity ratio $\mathrm{Mn} \mathrm{II}$ at $293.3 / \mathrm{Mn}$ II at $348.3 \mathrm{~nm}$ is also shown. In this case, since these lines do not belong to the same multiplet, the general theoretical treatment of Section 2.2 .4 should be applied. As predicted by the theory, the intensity ratio $\mathrm{Mn} \mathrm{II}$ at $293.3 / \mathrm{Mn}$ II at $348.3 \mathrm{~nm}$ indeed depends on the acquisition delay (especially in single pulse high energy measurements). Moreover, for the intensity ratio of lines belonging to different multiplets, a strong dependence on the experimental configuration used is evident. This behaviour is made clear considering the two limits of weak and strong selfabsorption (Eqs. (18) and (20)), represented in figure with dotted and dashed lines, respectively. The experimental results are coherent with a strong increase of the self-absorption of the lines changing the experimental conditions from the lowest laser energy (60 $\mathrm{mJ}$ single pulse) to the highest laser energy
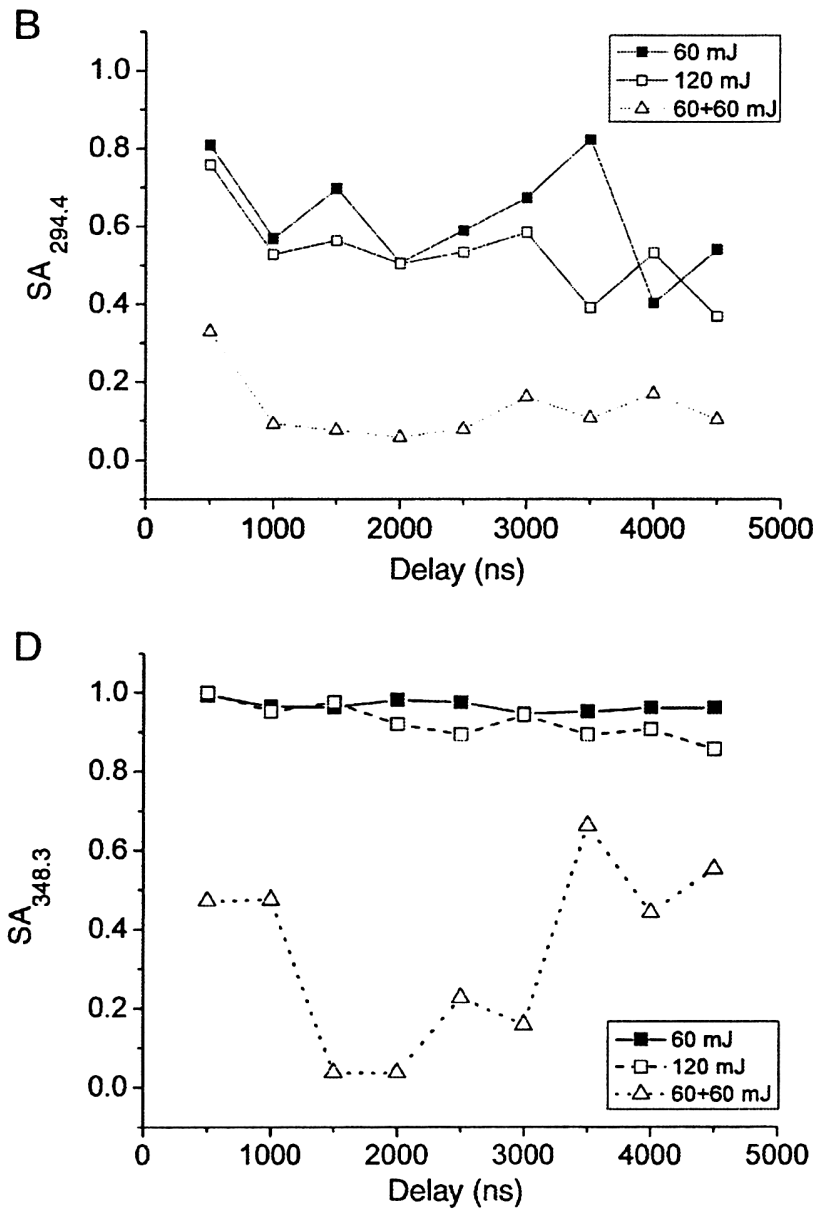

Fig. 6. Time dependence of the self-absorption coefficients SA for some of the Mn II lines considered in this paper (full squares, $60 \mathrm{~mJ}$ laser energy; open squares, $120 \mathrm{~mJ}$ laser energy; open triangles, 60+60 mJ double pulse measurements). A) Mn II at 293.3 nm, B) Mn II at 294.9 nm, C) Mn II at 346.0 nm, D) Mn II at 348.3 nm. 
(200 $\mathrm{mJ}$ single pulse) and finally to double pulse configuration $(60+60 \mathrm{~mJ})$. In this case the dependence of the intensity ratio on time delay practically coincides with the temperature dependence of the strong self-absorption limit at each time delay considered. The same considerations apply for the other line intensity ratios of the lines reported in Table 1 (not shown here). The opacities and therefore the SA coefficients of the Mn II lines here considered can be calculated using Eq. (25). Some results are reported in Fig. 6.

\subsection{Measurements on neutral Mn lines}

Seven Mn I lines have been considered for this study (Mn I at $382.4 ; 383.4 ; 471.0 ; 472.7 ; 473.9 ; 478.5 ; 482.4 \mathrm{~nm})$. Also in this case, the lines are well insulated and free from interferences of other Mn lines. The spectroscopic parameters of the lines considered are reported in Table 1. In Fig. 5, the intensity ratio of three neutral Mn lines (Mn I at $478.5 / \mathrm{Mn} \mathrm{I}$ at $482.4 \mathrm{~nm}$ and $\mathrm{Mn} \mathrm{I}$ at $482.4 / \mathrm{Mn} \mathrm{I}$ at $472.7 \mathrm{~nm}$ ) is reported, in single pulse configuration $(60 \mathrm{~mJ}$ and $120 \mathrm{~mJ})$ and double pulse configuration $(60+60 \mathrm{~mJ})$. Since the spectral windows used in the La Plata experiment did not include these Mn I lines, only the Pisa results will be shown here. The first couple of lines in Fig. 5 (Mn I at 478.5 and $\mathrm{Mn}$ I at $482.4 \mathrm{~nm}$ ) belongs to the same multiplet, so that the theoretical treatment of Section 2.2.3 is applicable. It is evident that the intensity ratio of these two lines is essentially independent on the acquisition delay. On the other hand, the low self-absorption limit predicted for these two lines $(0.8)$ is very close to the high self-absorption limit $(0.9)$, so that no appreciable difference can be observed, within the experimental error, between the measurements corresponding to different experimental configurations.

In the same figure, the intensity ratio of $\mathrm{Mn} \mathrm{I}$ line at $482.4 \mathrm{~nm}$ with $\mathrm{Mn}$ I line at $472.7 \mathrm{~nm}$ is also shown. In this case, since these lines do not belong to the same multiplet, the general theoretical treatment of Section 2.2.4 should be applied. In fact, the intensity ratio $\mathrm{Mn} \mathrm{I}$ at $482.4 / \mathrm{Mn}$ I at $472.7 \mathrm{~nm}$ depends, although weakly, on the acquisition delay, as expected from Eq. (25), due to the variation in plasma temperature with time.

As in the case of ionized Mn lines, the ratio of lines belonging to different multiplets shows a dependence on the experimental configuration, which is coherent with an increase of self-absorption with the increase of the laser energy.

The opacities and SA coefficients of the seven Mn I lines here considered can be calculated using Eq. (25); some relevant results are reported in Fig. 7.

\section{Discussion}

The variation of the self-absorption with the acquisition delay depends on the growth of plasma dimensions, on the decrease of plasma temperature and on the atoms-ions equilibrium, all the three effects being strictly interrelated during the plasma dynamic expansion. The increase of dimensions of the plasma affects the SA coefficient in the growth of the optical path $l$ and in the decrease of the number density $n \propto 1 / l^{3}$, the net effect being a reduction of self-absorption $\left(k(l) \cdot l \propto 1 / l^{2}\right)$. On the contrary, the cooling of the plasma tends to increase the population of the lower atomic energy levels in proportion to that of the higher levels and thus to enhance the self-absorption of the lines. On the other hand the plasma temperature variation leads also to a shift of the atoms-ions equilibrium, which is however strongly dependent on the plasma electron density according to the Saha equation.

It is clear from Figs. 6 and 7 that the SA coefficient at the different wavelengths decreases and then the plasma opacity dramatically increases in double pulse configuration, for both neutral and ionized Mn lines. In those conditions, the error in the determination of the absolute value of the plasma opacity can be quite high. In fact, although the treatment of self-absorption effects here presented is quite general, under the hypotheses of plasma homogeneity and Local Thermal Equilibrium, from an experimental point of view for obtaining a quantitative estimation of the line opacities the dependence of the right side of Eq. (25) on the $\kappa\left(\lambda_{0}\right)_{2} l$ parameter should be strong enough, for making the comparison with the experimental determined value
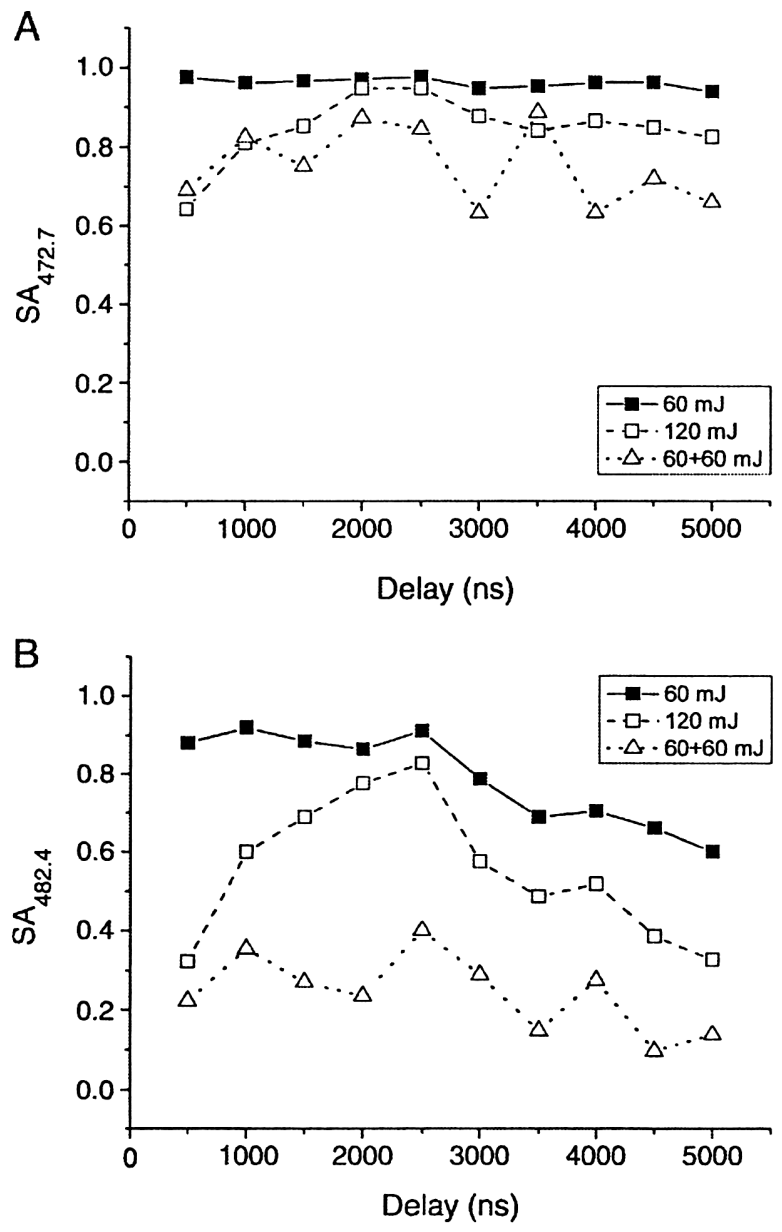

Fig. 7. Time dependence of the self-absorption coefficients SA for some of the Mn I lines considered in this paper (full squares, $60 \mathrm{~mJ}$ laser energy; open squares, $120 \mathrm{~mJ}$ laser energy; open triangles, $60+60 \mathrm{~mJ}$ double pulse measurements). A) Mn I at $472.7 \mathrm{~nm}$, B) Mn I at $482.4 \mathrm{~nm}$. 
of the line intensity ratio $\frac{\left(N_{\mathrm{p}}\right)_{2}}{\left(N_{\mathrm{p}}\right)_{1}}$ meaningful. However, we have already shown in Section 2.2.2 that Eq. (25) in the limit $\kappa\left(\lambda_{0}\right)_{2} l \gg>1$ transforms in

$\frac{\left(N_{\mathrm{p}}\right)_{2}}{\left(N_{\mathrm{p}}\right)_{1}}=a b^{\beta}$

i.e. the line intensity ratio (at a given temperature) becomes a constant. Therefore, the determination of the line opacity $\kappa\left(\lambda_{0}\right)_{2} l$ from the measurement of intensity ratio becomes impossible. As a consequence, the estimation of $\kappa\left(\lambda_{0}\right)_{2} l$ (and $\kappa\left(\lambda_{0}\right)_{1} l$ from Eq. (15)) can be obtained only in the limit of small to moderate selfabsorption $\left(\kappa\left(\lambda_{0}\right)_{2} l \leq 1\right)$. In the measurements performed in double pulse configuration, it is clear that the condition $\kappa\left(\lambda_{0}\right)$ $l \gg>1$ is realized at all time delays for all the lines here considered, therefore the only information we can obtain from the measurements is that all the $\mathrm{Mn}$ lines here considered are strongly self absorbed.

However, in single pulse configuration, at least for ionized Mn lines, the increase of plasma opacity with the acquisition delay, at both $60 \mathrm{~mJ}$ and $120 \mathrm{~mJ}$ laser energy, is well appreciable. Moreover, in the La Plata measurements a clear transition between the low self-absorption to high self-absorption regime is visible at increasing acquisition delays. Since in this condition of irradiation the plasma is strongly ionized $\left(n^{\mathrm{I}} /\left(n^{\mathrm{I}}+n^{\mathrm{II}}\right) \sim 0.1\right.$ during the time window investigated) and the increase in time of the plasma dimensions would tend to lower the opacity, on the basis of what discussed above it is clear that the increase of selfabsorption with the acquisition delay is justifiable by the cooling of the plasma.

In the present experimental conditions it is clear that the five Mn II lines belonging to the multiplet centred around $347.0 \mathrm{~nm}$ are considerably less self-absorbed than the corresponding lines at 293.3 and $294.9 \mathrm{~nm}$, by about one order of magnitude.

The acquisition delay dependence of the opacities of the Mn I lines considered is more complex and difficult to interpret, being also strongly affected by the atoms-ions equilibrium. It is possible that the initial growth of the self-absorption coefficient (corresponding to a decrease of the self-absorption effect) observed in Fig. 7 in the case of single pulse with energy $120 \mathrm{~mJ}$, is due to the strong decrease of atoms number density due to the initial fast expansion of the plasma. However, it is clear that at all the delays considered the Mn I lines belonging to the multiplet centred around $472 \mathrm{~nm}$ are definitely less self-absorbed than the $\mathrm{Mn} I$ lines at 478.5 and $482.4 \mathrm{~nm}$. For these lines the condition $\kappa\left(\lambda_{0}\right)_{2} l<<1$ is well realized, in single pulse configuration and at the energies used in the Pisa experiment, practically at all the time delays here considered. These lines can thus be good candidates for analytical measurements on $\mathrm{Fe}-\mathrm{Mn}$ alloys. The results reported above pose some questions on the use of double pulse LIBS method for the analysis of the major components of a given material; in fact, the strong improvement on signal intensity reported in double pulse configuration brings as a side effect a strong increase of the selfabsorption effect, which is very difficult to deal with, at the levels measured in the experimental conditions of this paper, in the framework of precise analytical measurements.

\section{Conclusions}

Starting from the basic equations describing the selfabsorption process in a homogeneous plasma, we have derived several analytical relations and devised experimental procedures for the evaluation of self-absorption coefficients (even in the absence of known Stark coefficients), which are important for characterization and control of the experimental conditions in which the analysis is performed. Although the experimental uncertainties and the strong starting hypotheses of the method in many cases do not allow a precise calculation of the selfabsorption coefficients, a quick and clear discrimination of the experimental conditions and the emission lines which are less sensitive to self-absorption, and thus are more appropriate for analytical measurements, can be obtained. This estimation, in our opinion, can be very useful in practice because it is fast and do not rely upon any external (not experimental) parameter such as plasma dimensions $l$, plasma number density or target composition. These results are considered as preliminary for the following study, which will be reported in a forthcoming paper, on matrix effects in LIBS analysis of FeMn alloys.

\section{References}

[1] D.A. Cremers, L.J. Radziemski, Detection of chlorine and fluorine in air by Laser-Induced Breakdown Spectrometry, Anal. Chem. 55 (1983) 1252-1256.

[2] L.J. Radziemski, D.A. Cremers, Laser-Induced Plasmas and Applications, Marcel Dekker Inc, New York, 1989.

[3] Y.L. Lee, K. Song, J. Sneddon, in: J. Sneddon, T.L. Thiem, Y.L. Lee (Eds.), Lasers in Analytical Atomic Spectroscopy, VCH Publishers Inc., 1997.

[4] D.A. Cremers, L.J. Radziemski, T.R. Loree, Spectrochemical analysis of liquids using the laser spark, Appl. Spectrosc. 38 (1984) 721-729.

[5] D. Bulajic, G. Cristoforetti, M. Corsi, M. Hidalgo, S. Legnaioli, V. Palleschi, A. Salvetti, E. Tognoni, S. Green, D. Bates, A. Steiger, J. Fonseca, J. Martins, J. McKay, B. Tozer, D. Wells, R. Wells, M.A. Harith, Diagnostics of high-temperature steel pipes in industrial environment by Laser-Induced Breakdown Spectroscopy technique: the LIBSGRAIN project, Spectrochim. Acta Part B 57 (2002) 1181-1192.

[6] A.K. Ray, F.Y. Yueh, J.P. Singh, Laser-Induced Breakdown Spectroscopy of molten aluminium alloy, Appl. Opt. 42 (2003) 2078-2084.

[7] S. Lawson, C.P. Evans, A.I. Whitehouse, J. Wright, J. Young, LIBS laser eyes for peering into the radioactive zone, Mater. Word 8 (2000) 5 .

[8] A.I. Whitehouse, J. Young, I.M. Botheroyd, S. Lawson, C.P. Evans, J. Wright, Remote material analysis of nuclear power station steam generator tubes by Laser-Induced Breakdown Spectroscopy, Spectrochim. Acta Part B 56 (2001) 821-830.

[9] A. Ciucci, V. Palleschi, S. Rastelli, R. Barbini, F. Colao, R. Fantoni, A. Palucci, S. Ribezzo, H.J.L. van der Steen, Trace pollutants analysis in soil by a time resolved Laser-Induced Breakdown Spectroscopy technique, Appl. Phys., B 63 (1996) 185-190.

[10] S.G. Buckley, H.A. Johnsen, K.R. Henken, D.W. Hahn, Implementation of Laser-Induced Breakdown Spectroscopy as a continuous emission monitor for toxic metals, Waste Manage. 20 (2000) 455-462.

[11] R.W. Bogue, Boom time for LIBS Technology, Sens. Rev. 24 (2004) 353-357.

[12] I.V. Cravetchi, M. Taschuk, G.W. Rieger, Y.Y. Tsui, R. Fedosejevs, Spectrochemical microanalysis of aluminium alloys by Laser-Induced Breakdown Spectroscopy: identification of precipitates, Appl. Opt. 42 (2003) 6138-6147.

[13] S. Palanco, L.M. Cabalin, D. Romero, J.J. Laserna, Infrared laser ablation and atomic emission spectrometry of stainless steel at high temperature, J. Anal. At. Spectrom. 14 (1999) 1883-1887. 
[14] V. Sturm, L. Peter, R. Noll, Steel analysis with Laser-Induced Breakdown Spectrometry in the Vacuum ultraviolet, Appl. Spectrosc. 54 (2000) 1275-1278.

[15] H. Bette, R. Noll, High speed Laser-Induced Breakdown Spectrometry for scanning microanalysis, J. Phys., D, Appl. Phys. 37 (2004) 1281-1288.

[16] J. Gruber, J. Heitz, N. Arnold, D. Bauerle, N. Ramaseder, W. Meyer, J. Hochortler, F. Kock, In situ analysis of metal melts in metallurgic vacuum devices by Laser-Induced Breakdown Spectroscopy, Appl. Spectrosc. 58 (2004) 457-462.

[17] G.P. Kopitsa, V.V. Runov, S.V. Grigoriev, V.V. Bliznuk, V.G. Gavriljuk, N.I. Glavatska, The investigation of $\mathrm{Fe}-\mathrm{Mn}$-based alloys with shape memory effect by small-angle scattering of polarized neutrons, Physica. B + C 335 (2003) 134-139.

[18] T. Schneider, M. Acet, B. Rellinghaus, E. Wassermann, W. Pepperhoff, Antiferromagnetic Invar and anti-Invar in Fe-Mn alloys, Phys. Rev., B 51 (1995) 8917-8921.

[19] A. Borgenstam, M. Hillert, The driving force for Lath and Plate martensite and the activation energy for isothermal martensite in ferrous alloys, J. Phys., IV 7 (1997) C5:23-C5:28.

[20] S.M. Cotes, A. Fernández Guillermet, M. Sade, Gibbs energy modelling of the driving forces and calculation of the fcc/hcp martensitic transformation temperatures in Fe-Mn and Fe-Mn-Si alloys, Mater. Sci. Eng., A Struct. Mater.: Prop. Microstruct. Process. 273-275 (1999) 503-506.

[21] A. Baruj, A. Fernández Guillermet, M. Sade, Effects of thermal cycling and plastic deformation upon the Gibbs energy barriers to martensitic transformation in Fe-Mn and Fe-Mn-Co alloys, Mater. Sci. Eng., A Struct. Mater.: Prop. Microstruct. Process. 273-275 (1999) 507-511.

[22] I.B. Gornushkin, J.M. Anzano, L.A. King, B.W. Smith, N. Omenetto, J.D. Winefordner, Curve of growth methodology applied to laser-induced breakdown spectroscopy, Spectrochim. Acta Part B 54 (1999) 491-503.

[23] C. Aragón, J. Bengoechea, J.A. Aguilera. Influence of the optical depth on spectral line emission from laser-induced plasmas, Spectrochim. Acta Part B 56 (2001) 619-628.

[24] H. Amamou, A. Bois, B. Ferhat, R. Redon, B. Rossetto, M. Ripert, Correction of the self-absorption for reversed spectral lines: application to two resonance lines of neutral aluminum, J. Quant. Spectrosc. Radiat. Transfer 77 (2003) 365-372.

[25] I.B. Gornushkin, C.L. Stevenson, B.W. Smith, N. Omenetto, J.D. Winefordner, Modeling an inhomogeneous optically thick laser induced plasma: a simplified theoretical approach, Spectrochim. Acta Part B 56 (2001) 1769-1785.

[26] J.A. Aguilera, J. Bengoechea, C. Aragón, Curves of growth of spectral lines emitted by a laser-induced plasma: influence of the temporal evolution and spatial inhomogeneity of the plasma, Spectrochim. Acta Part B 58 (2003) 221-237.

[27] D. Bulajic, M. Corsi, G. Cristoforetti, S. Legnaioli, V. Palleschi, A. Salvetti, E. Tognoni, A procedure for correcting self-absorption in calibration free-laser induced breakdown spectroscopy, Spectrochim. Acta Part B 57 (2002) 339-353.

[28] A.M. El Sherbini, Th.M. El Sherbini, H. Hegazy, G. Cristoforetti, S. Legnaioli, V. Palleschi, L. Pardini, A. Salvetti, E. Tognoni, Evaluation of
Self-absorption Coefficients of Aluminum Emission Lines in LaserInduced Breakdown Spectroscopy Measurements, Spectrochim. Acta Part B 60 (2005) 1573-1579.

[29] A. Ciucci, V. Palleschi, S. Rastelli, A. Salvetti, E. Tognoni, New procedure for quantitative elemental analysis by Laser Induced Plasma Spectroscopy, Appl. Spectrosc. 53 (1999) 960-964.

[30] H. Hakkanen, J. Korppi-Tommola, Laser-induced plasma emission spectrometric study of pigments and binders in paper coatings: matrix effects, Anal. Chem. 70 (1998) 4727-4736.

[31] J.M. Anzano, I.B. Gornushkin, S.I. Gornushkin, B.W. Smith, J.D. Winefordner, Effective normalization technique for correction of matrix effects in Laser-induced Breakdown Spectroscopy detection of magnesium in powdered samples, Appl. Spectrosc. 56 (2002) 433-436

[32] S. Djenize, S. Bukvic, A. Sreckovic, Z. Nikolic, The first measured Mn II and Mn III Stark broadening parameters, New Astron. 11 (2006) 256-261.

[33] L.C. Popovic, M.S. Dimitrijevic, The electron impact broadening parameters in hot star atmospheres: Mn II, Mn III, Ga III, Ge III and Ge IV lines, Astron. Astrophys., Suppl. Ser. 128 (1998) 203-205.

[34] F. Colao, R. Fantoni, V. Lazic, A. Paolini, LIBS application for analyses of martian crust analogues: search for the optimal experimental parameters in air and $\mathrm{CO}_{2}$ atmosphere, Appl. Phys., A 79 (2004) 143-152.

[35] H. Amamou, A. Bois, B. Ferhat, R. Redon, B. Rossetto, P. Matheron, Correction of self-absorption spectral line and ratios of transition probabilities for homogeneous and LTE plasma, J. Quant. Spectrosc. Radiat. Transfer 75 (2002) 747-763.

[36] M. Friedjung, G. Muratorio, Singly ionized iron as a diagnostic of stellar envelopes. 1. The methods, Astron. Astrophys. 188 (1987) 100

[37] S.O. Kastner, Clarification and interpretation of the Friedjung-Muratorio self absorption curve method. Astron. Astrophys. 351 (1999) 1016-1020.

[38] S. Chandrasekar, Radiative Transfer, Dover Publications, Inc., New York, 1960.

[39] R.S. Adrain, J. Watson, Laser microspectral analysis: a review of principles and applications, J. Phys., D, Appl. Phys. 17 (1984) 1915-1940.

[40] H.R. Griem, Plasma Spectroscopy, Mc Graw Hill, New York, 1964.

[41] G. Cristoforetti, S. Legnaioli, V. Palleschi, L. Pardini, A. Salvetti, E. Tognoni, Modi: a new mobile instrument for in situ standardless LIBS analysis of Cultural Heritage, in: R. Salimbeni, L. Pezzati (Eds.), Optical Methods for Arts and Archaeology, Proc. SPIE, vol. 5857, 2005, pp. 129-138.

[42] J. Ashkenazy, R. Kipper, M. Caner, Spectroscopic measurements of electron density of capillary plasma based on Stark broadening of hydrogen lines, Phys. Rev., A 43 (1991) 5568-5574.

[43] S. Yalcin, D.R. Crosley, G.P. Smith, G.W. Faris, Influence of ambient conditions on the laser air spark, Appl. Phys., B 68 (1999) 121-130.

[44] M. Corsi, G. Cristoforetti, M. Giuffrida, M. Hidalgo, S. Legnaioli, V. Palleschi, A. Salvetti, E. Tognoni, C. Vallebona, Three-dimensional analysis of laser induced plasmas in single and double pulse configuration, Spectrochim. Acta Part B 59 (2004) 723-735.

[45] G. Cristoforetti, S. Legnaioli, V. Palleschi, A. Salvetti, E. Tognoni, P. Tomassini, Reconstruction of laser-induced plasma spectral emissivity in non-axisymmetric conditions, Spectrochim. Acta Part B 60 (2005) 888-896. 